\title{
BMJ Open The barriers to and enablers of providing reasonably adjusted health services to people with intellectual disabilities in acute hospitals: evidence from a mixed-methods study
}

\author{
Irene Tuffrey-Wijne, ${ }^{1}$ Lucy Goulding, ${ }^{2}$ Nikoletta Giatras, ${ }^{2}$ Elisabeth Abraham, ${ }^{2}$ \\ Steve Gillard, ${ }^{2}$ Sarah White, ${ }^{2}$ Christine Edwards, ${ }^{3}$ Sheila Hollins ${ }^{2}$
}

To cite: Tuffrey-Wijne I, Goulding L, Giatras N, et al. The barriers to and enablers of providing reasonably adjusted health services to people with intellectual disabilities in acute hospitals: evidence from a mixedmethods study. BMJ Open 2014:4:e004606. doi:10.1136/bmjopen-2013004606

- Prepublication history and additional material is available. To view these files please visit the journal online (http://dx.doi.org/10.1136/ bmjopen-2013-004606).

Received 3 December 2013 Revised 13 February 2014 Accepted 21 February 2014

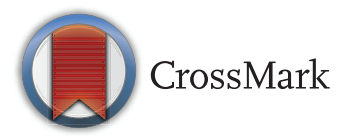

For numbered affiliations see end of article.

Correspondence to Dr Irene Tuffrey-Wijne; I.Tuffrey-Wijne@sgul. kingston.ac.uk

\section{ABSTRACT}

Objective: To identify the factors that promote and compromise the implementation of reasonably adjusted healthcare services for patients with intellectual disabilities in acute National Health Service (NHS) hospitals.

Design: A mixed-methods study involving interviews, questionnaires and participant observation (July 2011March 2013).

Setting: Six acute NHS hospital trusts in England. Methods: Reasonable adjustments for people with intellectual disabilities were identified through the literature. Data were collected on implementation and staff understanding of these adjustments.

Results: Data collected included staff questionnaires $(n=990)$, staff interviews $(n=68)$, interviews with adults with intellectual disabilities $(n=33)$, questionnaires $(n=88)$ and interviews $(n=37)$ with carers of patients with intellectual disabilities, and expert panel discussions $(n=42)$. Hospital strategies that supported implementation of reasonable adjustments did not reliably translate into consistent provision of such adjustments. Good practice often depended on the knowledge, understanding and flexibility of individual staff and teams, leading to the delivery of reasonable adjustments being haphazard throughout the organisation. Major barriers included: lack of effective systems for identifying and flagging patients with intellectual disabilities, lack of staff understanding of the reasonable adjustments that may be needed, lack of clear lines of responsibility and accountability for implementing reasonable adjustments, and lack of allocation of additional funding and resources. Key enablers were the Intellectual Disability Liaison Nurse and the ward manager.

Conclusions: The evidence suggests that ward culture, staff attitudes and staff knowledge are crucial in ensuring that hospital services are accessible to vulnerable patients. The authors suggest that flagging the need for specific reasonable adjustments, rather than the vulnerable condition itself, may address some of the barriers. Further research is recommended that

\section{Strengths and limitations of this study}

- This is the largest study to date focusing on reasonable adjustments in hospital care for people with intellectual disabilities. Employing both qualitative and quantitative methods and triangulation of data from a wide range of data sources, including patients and carers as well as clinical staff and strategic hospital managers, strengthens the findings.

- Hospital trusts across England had been publicly named in connection with poor care provision to patients with intellectual disabilities, which may have led to a desire by the participating trusts and staff to be seen in a positive light with regard to caring for people with intellectual disabilities, and to under-reporting of any problems or concerns.

- The number of carers and people with intellectual disabilities participating in the study was relatively small in relation to staff participants (although the sample size was large in comparison with existing studies, and saturation of data has been achieved).

- Sampling of patients and carers was facilitated by the Intellectual Disability Liaison Nurse (IDLN) or Intellectual Disability Lead at each study site, leading to sampling bias and a difficulty in accessing a sample of patients and carers who had no involvement from the IDLN.

- The research team had no access to a sample of patients who had not been identified or flagged as having intellectual disabilities.

describes and quantifies the most frequently needed reasonable adjustments within the hospital pathways of vulnerable patient groups, and the most effective organisational infrastructure required to guarantee their use, together with resource implications. 


\section{BACKGROUND}

An estimated $2 \%$ of the population have intellectual disabilities (in the UK also known as 'learning disabilities'). ${ }^{1}$ There have been a number of research studies, reports and government-commissioned inquiries around the health inequalities experienced by people with intellectual disabilities in the UK. ${ }^{2-8}$ These have consistently highlighted poor healthcare provision for this population, leading to avoidable harm and premature, avoidable deaths. ${ }^{2}{ }^{9}$ This has led to a range of recommendations and strategies for promoting better and safer healthcare delivery to people with intellectual disabilities. Among these recommendations has been a consistent emphasis on the need to focus on the effective delivery ${ }^{4}$ and audit ${ }^{2}$ of 'reasonably adjusted' health services to people with intellectual disabilities, along with descriptions of what kind of reasonable adjustments may be needed by this patient group.

It is a legal requirement in England for public services to ensure that their services are adjusted in order to make them accessible to people with disabilities. ${ }^{10}{ }^{11}$ In practice, making reasonable adjustments means doing things differently to normal to ensure that people with intellectual disabilities are not disadvantaged. ${ }^{12}$ An ongoing national survey of National Health Service (NHS) trusts has found many examples of good practice, with some forms of reasonable adjustment being widely adopted in some trusts. ${ }^{13}$ The most commonly reported was the provision of more accessible information. Trusts also reported a reliance on specialist staff with intellectual disability expertise to act in liaison roles with regard to face-to-face contact with patients with intellectual disabilities.

However, despite a clear legal framework, and despite examples of good practice, various investigations and inquiries have noted that comprehensive reasonable adjustments are not routinely provided and health services often remain inaccessible to people with intellectual disabilities, compromising patient safety. ${ }^{8}{ }^{13-16}$ In 2008, the Department of Health (England) stated that progress on health inequalities will be judged against how public services treat especially vulnerable groups, and that 'if services and health outcomes are improving for people with learning disabilities, they are likely to be improving for other groups at risk of health inequalities' (ref 17, p.6). The provision of safe, reasonably adjusted healthcare services for people with intellectual disabilities can therefore be used as a benchmark for the care and treatment of all vulnerable patients within acute hospitals.

Given the evidence that a failure to provide effective reasonable adjustments leads to premature deaths among patients with intellectual disabilities, ${ }^{2}$ there is an urgent need to understand why, despite national recommendations and a legal duty, the provision of reasonable adjustments has not been consistent and successful. This study aimed to describe the cross-organisational, organisational and individual factors that facilitate or compromise the safety of patients with intellectual disabilities in NHS acute hospitals. The full findings are described elsewhere. ${ }^{18}$ This paper reports on the findings in relation to the following research question: 'What are the barriers to providing reasonably adjusted health services to patients with intellectual disabilities in NHS acute hospitals?'

\section{STUDY METHODS \\ Design}

This was a mixed-methods study in three stages, involving interviews and questionnaire surveys (July 2011March 2013). Stage I was focused on mapping the study sites' systems and structural changes in response to the requirement to provide reasonably adjusted health services for patients with intellectual disabilities. Stage II involved examining the implementation of reasonable adjustments at ward/clinic level. Stage III involved a structured discussion of emerging findings with expert panels of strategic hospital managers and senior clinicians at the study sites.

\section{Setting}

The study was conducted at six NHS acute hospital trusts in England. The sites were purposively selected to cover a range of different sizes, demographic areas and models for accessing intellectual disability expertise (see table 1).

\section{Participants}

The total number of participants was 1251; a breakdown is given in online supplementary appendix 1 . The study collaborator at each site (either the director or deputy director of nursing, or the on-site Intellectual Disability Liaison Nurse (IDLN)) facilitated recruitment.

Online questionnaires were sent to all clinical hospital staff with a caseload of patients.

Face-to-face semi-structured interviews $(n=68)$ were conducted with senior hospital managers (including the medical director and director of nursing at each site) and purposively selected clinical staff from two or three hospital wards per site. Selection criteria specified that these wards should be a medical assessment unit or similar, a ward selected by the collaborator as having a relatively high number of patients with intellectual disabilities and the ward that had received the highest number of complaints (not restricted to complaints about people with intellectual disabilities). On each selected ward, the ward manager or sister was interviewed and asked to select two further ward nurses for interview. In addition, staff members with specific responsibilities for implementing intellectual disability policies were purposively selected for interview.

Semi-structured face-to-face interviews were conducted with a convenience sample of adults with intellectual disabilities who had used the hospital; recruitment was facilitated by an IDLN or community intellectual disability nurse $(n=33)$. The participant's ability to give informed consent was assessed by the researchers both 
Table 1 Description of participating NHS hospital trusts

\begin{tabular}{llll}
\hline Hospital & Type & Area & Intellectual disability liaison nurse \\
\hline A & Teaching & Urban & Hospital based \\
B & District general & Urban & Community based \\
C & District general & Urban & None \\
D & District general & Urban/rural & Hospital based \\
E & Teaching & Urban/rural & Hospital based \\
F & District general & Rural & Community based \\
\hline
\end{tabular}

through communication with the participant prior to interview and, where appropriate, liaison with ID professionals or carers. Prospective participants were given easy-read, pictorial study information. The study was explained, supported by this material, prior to the participant giving consent. Special attention was paid to ensuring that the researchers were sensitive to the various ways in which people with intellectual disabilities may express withdrawal of consent.

Postal questionnaires were sent to family carers and paid carers of patients with intellectual disabilities who had been a patient during a 12-month period (usable questionnaires returned: $n=88$ ). Semi-structured face-toface and telephone interviews were held with a selfselected sample of carer survey respondents $(n=37)$.

Expert panels consisted of purposively selected staff at four sites; the collaborators were asked to invite the hospital's senior managers and senior clinicians who had a responsibility for patients with learning disabilities or other specific vulnerable patient groups $(n=42)$.

\section{Data collection tools}

The study was underpinned by a conceptual research framework on patient safety, derived from the literature on healthcare for people with intellectual disabilities and organisational change (see online supplementary appendix 2). This conceptual framework informed the development of the interview schedules and questionnaires. They included questions on the availability and implementation of the reasonable adjustments needed by people with intellectual disabilities as identified in the literature (see online supplementary appendix 3).

Respondents to the staff questionnaire were asked to indicate on a Likert-type scale to what extent there was flexibility within their clinical setting to provide such adjustments. Questions were included on: the identification of patients with intellectual disabilities within the respondents' clinical areas, any training the staff had received, the provision of specific reasonable adjustments within the respondents' clinical areas (as identified in the literature) and staff attitudes towards reasonable adjustments.

The staff interview schedule included the following questions: 'What adjustments or changes to normal practice do you think might need to be made so that people with intellectual disabilities can receive the healthcare they need? Do you have any real life examples of when you have made these adjustments? Can you think of any other specific needs of patients with intellectual disabilities? Is your ward able to meet these needs?'

Carers were asked Likert-type scale questions about the extent to which reasonable adjustments had been implemented during the most recent hospital episode of the person with intellectual disabilities. They were also asked to list the person's special needs or additional difficulties, and to indicate on a Likert-scale how well the hospital met this need.

People with intellectual disabilities were presented with pictures, pictorial stories and/or straightforward questions, according to their receptive and expressive communication abilities. They were asked to comment on their hospital experiences with a specific focus on adjustments, such as being given sufficient time and additional resources if needed. Researchers with intellectual disabilities were partnered with researchers without intellectual disabilities to cofacilitate these interviews.

\section{Data analysis}

This was a complex multimethod study involving the collection of qualitative and quantitative data from six sites. An analytical framework was derived from the conceptual framework underpinning the study, and was used to analyse and synthesise findings from across data sets. The purpose of this was to develop an empirical framework representing the barriers and enablers of providing safe, reasonably adjusted healthcare services to people with intellectual disabilities. The analytical framework informed a number of analytical questions that were asked of the data (see online supplementary appendix 3). All data from across data sets were examined for concordance and convergence with the framework. This was supported by the data management programme QSR NVivo 9. Analysis was undertaken with involvement from all members of the multidisciplinary team. This involved coding subsamples of the data and weekly discussions with the core research team to decide possible new analytical codes. There was an ongoing analytic strategy allowing for refinement of the analytic framework with emerging findings and possible new analytical categories. Reliability was enhanced by involving members of the wider research team approximately once a month to discuss data sets that did not fit into the framework or were difficult to synthesise. Meetings were also held with specific stakeholder representatives on the research advisory board, and with outside experts, to discuss emerging 
topics within their area of expertise. A final analytical framework was agreed in the final month of stage II.

Analysis of the qualitative data involved line-by-line coding and questionnaires were analysed using IBM SPSS Statistics V.19. Descriptive statistics were calculated and results were fed back into the development of the framework.

Specific attention was applied to identifying positive examples, where reasonable adjustments were made that met the needs of individual patients, and negative examples, where reasonable adjustments that may have made the hospital service more accessible for the patient were not provided. These examples were scrutinised with a focus on determining the factors that underpinned them. Examples of such refinement are the inclusion in the analytical framework of staff understanding of the Mental Capacity Act; the influence of ward managers, matrons or IDLNs who act as intellectual disability champions and the variability of the provision of reasonably adjusted services both within and between hospital wards.

\section{Ethical issues and approvals}

A few participants with intellectual disabilities who took part in the observation lacked capacity to consent to participate in the study; in these cases, the guidelines in the Mental Capacity Act $^{19}$ were followed. The Research Advisory Board included people with intellectual disabilities, family carers, practitioners and academics with extensive experience of working with people with intellectual disabilities in all research stages to ensure relevance, insight and sensitivity, especially with regard to ethical issues.

\section{RESULTS}

The findings reported here have been derived from across data sets. An empirical framework representing the full list of barriers and enablers in relation to providing reasonably adjusted health services for patients with intellectual disabilities is given in figure 1 . This reflects the final analytical framework, which has emerged from the data and therefore moves on from the original conceptual framework (see online supplementary appendix 2 ).

A total of 990 usable staff questionnaires were returned. Approximate response rates at the six sites were $3 \%, 4 \%, 4 \%, 5 \%, 7 \%$ and $15 \%$. These were calculated based on the numbers of clinical staff working at each hospital; variations are likely to be due to differences in the definition of 'clinical staff' and different methods of distribution, with some sites taking a more targeted approach than others. Selected findings from the staff questionnaire are given in figure 2.

Senior strategic managers were aware of the legal duty and their responsibility to provide reasonable adjustments. All six study sites had certain systems in place which aimed to facilitate the delivery of reasonably adjusted health services for people with intellectual disabilities. This included strategies for accessing intellectual disability expertise, the introduction of patient-held information documents and policies to support carers. A wide range of positive examples of reasonable adjustments were found at all six study sites (see box 1).

However, the application of reasonable adjustments 'on the ground', including those that had been introduced at policy level, was not consistent. The extent to which health services were reasonably adjusted varied not only within hospital sites but also within wards and clinical

\section{Barriers}

Enablers

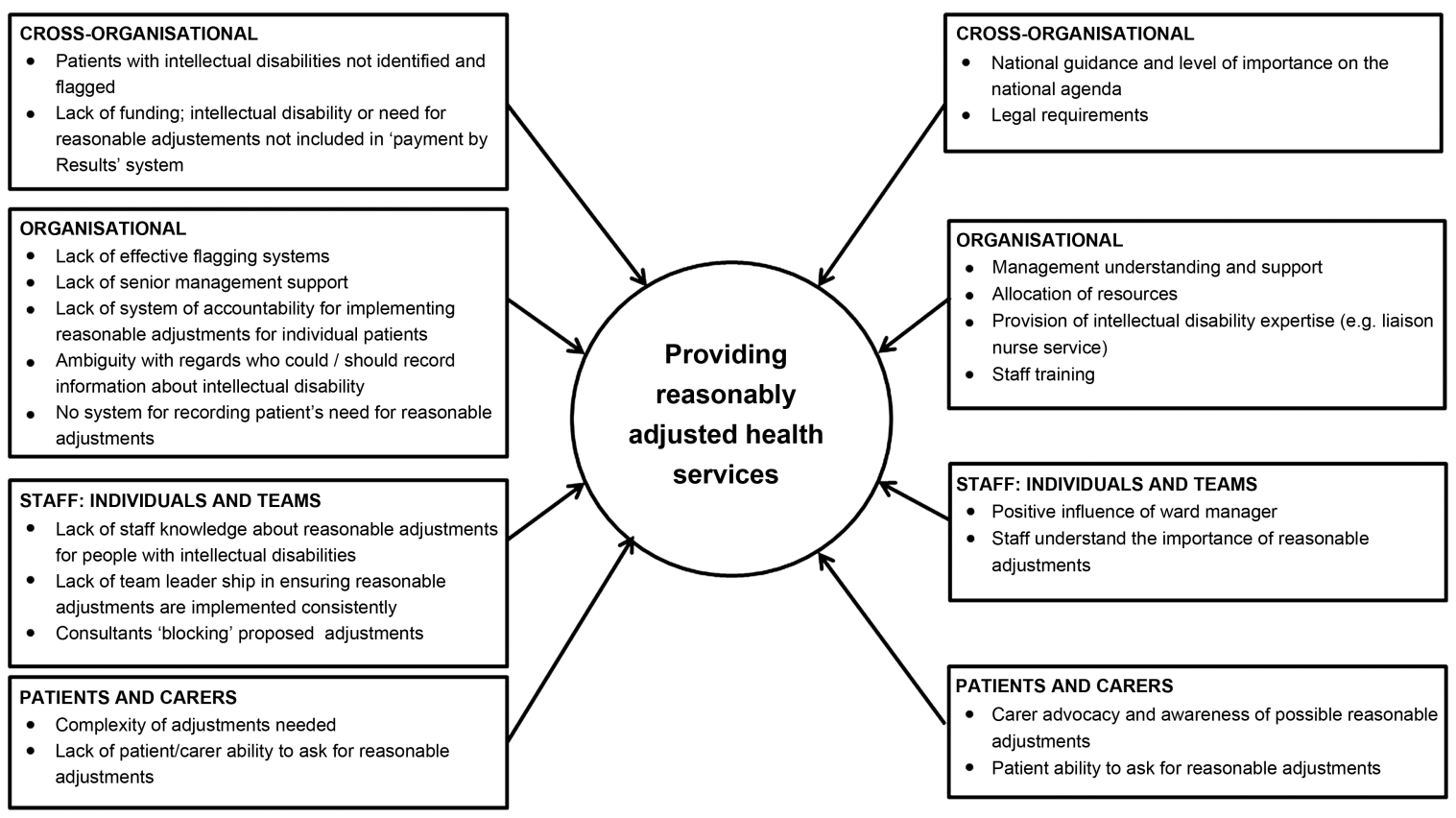

Figure 1 Empirical framework: barriers and enablers in relation to provide reasonably adjusted health services to patients with intellectual disabilities. 
Figure 2 Selected findings from staff questionnaire in response to the question: 'When caring for a patient with intellectual disabilities, is there flexibility in your clinical setting to' $(n=825)$. Values in percentages.

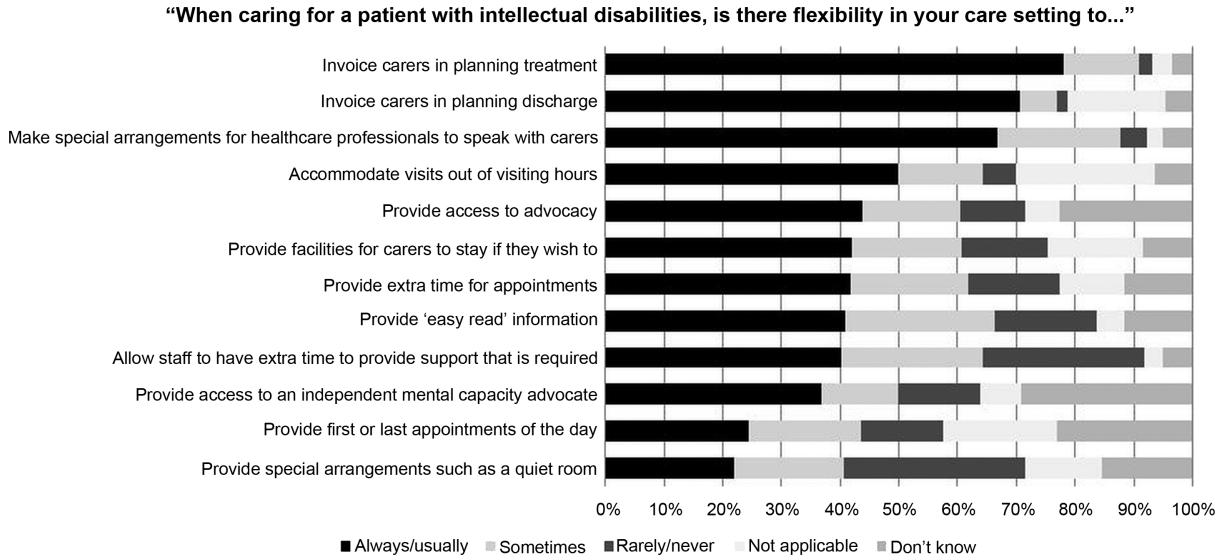

- Always/usually $\approx$ Sometimes Rarely/never Not applicable Don't know

how they find out this information, they answered that they were most commonly alerted by colleagues $(72 \%$, 710 of 987$)$, through the patient's notes $(65 \%, 641$ of 987 ) or told by the patient or the patient's carer $(57 \%$, 564 of 987 ); $13 \%$ (131 of 987) were alerted through a computerised flagging system. Staff at outpatient departments was most likely to be uninformed of a patient's intellectual disability $(17 \%$, as opposed to $3 \%$ for inpatients staff). This meant that hospital staff were often unaware that reasonable adjustments might be needed until the patient arrived at the clinic, ward or department. There was also a lack of effective communication about known intellectual disability within the hospital structures, with failures to pass on information as patients moved between admissions, wards and across inpatient and outpatient departments. The effect was that reasonable adjustments, particularly those that needed advance planning, often could not be delivered, as was the case in the following example:

Radiographer (questionnaire): Frequently when booking appointments, we are not informed that patients have intellectual disabilities and doctors will request scans which when the patient arrives to have, it is immediately clearly completely inadequate for such a patient to be able to cope with the scan requested and therefore has to be abandoned (...) Were it to be made known to the imaging staff that the patient had intellectual disabilities, allowances could have been made before the time of the appointment to cater for them.

\section{Lack of staff understanding of the reasonable adjustments that may be needed}

There was widespread reluctance among staff to identify and flag patients with intellectual disabilities. This seemed to stem mostly from a belief that 'equal treatment' means 'the same treatment'. Staff at all levels of seniority wondered whether 'labelling' patients as having intellectual disabilities would make any difference to their treatment and care:

Interviewer: 'If you found out that a patient had a learning disability, is it compulsory on this ward to record it anywhere?' 
Box 1 A selection of examples of reasonably adjusted health services

Description of adjustment

A. Intellectual disability liaison nurses (IDLNs) provided training for hospital staff.

B. Patients with intellectual disabilities were provided with patient-held information documents where they could record key information for the benefit of hospital staff, including likes and dislikes.

C. Patients with intellectual disabilities and their carers who attended outpatient appointments were given a bleep, so they did not have to wait in the small waiting area.

D. A patient with intellectual disabilities who needed dental treatment but could not bear to come into the hospital building was sedated in the car park (with his consent and his family's support).

E. A patient with intellectual disabilities was offered a preadmission visit to look around the ward area.

F. A patient with intellectual disabilities could not cope with tests and treatments. When he came in for dental surgery under general anaesthetic, several other necessary tests and treatments were carried out at the same time, involving a range of different clinicians.

G. Carers were offered food, a bed and a parking permit.

$\mathrm{H}$. A woman with intellectual disabilities liked her consultant and responded well to him. When she was offered her next appointment with a different doctor, her mother asked whether she could see the same consultant. The consultant rearranged his schedule so he would always be the doctor to see this patient.

I. A Medical Assessment Unit ensured that patients with intellectual disabilities were moved rapidly to the relevant ward.

J. Another Medical Assessment Unit allowed patients with intellectual disabilities who had got used to the staff and who only needed a few days in hospital, to stay on the ward rather than be moved to a different ward.

K. Patients with intellectual disabilities who had significant care needs were allocated additional care staff by the hospital.

L. Patients with intellectual disabilities were allocated a quiet waiting area.

M. Patients with intellectual disabilities were given an early morning appointment and/or a double appointment.

N. A patient with intellectual disabilities who found it difficult to cope with a busy ward environment needed treatment on a day surgery ward where no separate room was available. She was given a bed by the window with the curtains pulled round. Staff told her exactly what to expect, and she coped well with the treatment and the environment.

0 . The carers of patients with intellectual disabilities were invited to attend the consultant's ward rounds.

Staff nurse: 'I don't know... I don't see how it would affect their nursing-how we give them their care. It wouldn't make any difference if they've got a learning disability or not.

Deputy director of nursing (interview): 'I have to question why we are labelling people, whether it's with a learning disability or with diabetes or with dementia-what's the purpose of us knowing?'
Box 2 A selection of examples of health services that were not reasonably adjusted

Description of lack of adjustment

A. Members of the staff team who provided care for a patient at home continued to provide the patient with support during his hospital stay. They were asked by ward staff to leave at the end of visiting hours.

B. A patient with intellectual disabilities who needed specialist dental treatment could not cope with the busy environment where he was asked to wait behind a screen. His family took him outside when he started self-harming and pulling at the screen. They asked to be given time to help the patient calm down, and to be seen in a quiet room. The reception staff refused. (This situation was redeemed when the family found a room to take the patient to, where the dentist came to find them; the dentist then suggested proceeding with the treatment based on a visual assessment, without doing an X-ray which would have made the whole procedure too long and unbearable for this patient.)

C. One ward nurse refused to give carers a parking permit on the basis that only families of terminally ill patients were entitled to this; another nurse on the same ward gave the carers a parking permit without hesitation.

D. Doctors and nurses did not give a patient with intellectual disabilities enough time.

E. A patient with intellectual disabilities, who was unable to give informed consent, was sent for a colonoscopy by his general practitioner, based on a 'best interest' decision. ${ }^{19}$ Hospital staff refused to perform the colonoscopy, because he could not give consent.

F. Patient-held information documents were filled in by carers but were not used by hospital staff.

G. A family carer had explained to hospital staff that the patient could not read or write, although she could sign her name. Despite this, the patient was given treatment consent forms to sign, without further explanations. She was also given written menus and asked to fill in her food choices, without support. Her failure to complete the menus led to the provision of inappropriate food.

In addition, senior managers said that flagging of intellectual disabilities would only be useful if staff subsequently knew how to adjust the individual patient's care:

Director of nursing (interview): 'One of the other flags is around infections, MRSA, and the staff immediately go into 'right, well that means they need to be in a side room, they need to do this, this and this'. So I guess what we need, is along with that flag, that flag means that the patient needs this, this and this, and if they've got those things well all well and good, if they haven't got those things then you need to put those in.'

There was a lack of understanding among staff that reasonable adjustments require, by law, that people are treated differently because of their different needs, and that this is necessary in order to provide people with intellectual disabilities with equal access to hospital services. Staff members' perceptions of the actions required 
Table 2 Types of reasonable adjustments that were important to many people with intellectual disabilities

\section{Type of reasonable adjustment \\ Adjusting communication, taking into account the patient's} receptive and expressive verbal capacity

Addressing the patient's ability to cope with different environments, changes in routine, unfamiliar procedures and unfamiliar staff

Addressing the patient's need to change ways in which care/ treatment is given

Including and supporting the patient's carers as expert care partners

Providing advocacy and support for mental capacity

Providing access to intellectual disability expertise

\section{Quotes}

Carer (questionnaire): 'Things were not explained to the patient in a way she could understand. Was confused and frustrated.'

Carer (questionnaire): 'Staff allowed patient time to process information and provided additional easy to understand explanations of condition and exercise programme.'

Person with intellectual disabilities (interview): 'l'm not sure whether I'm going to be seen; sometimes-wait, wait, wait, wait, ages, ages, ages, ages to get seen'

Consultant anaesthesist (questionnaire): Learning disability patients are dealt with on dedicated lists. They are given side rooms if done in day surgery. If they are on a main operating list they always go first. Their carers are allowed to be with them apart from in theatre. No visiting times apply' Carer (questionnaire): 'The nurse said [patient] cannot be screened because she cannot sit up by herself and hold onto anything, therefore the screening cannot be done' Carer (interview): 'We went to [name of hospital], it was for an x-ray (...) I said we would need a hoist (...) So I called [the hospital's IDLN] and she met us there. She said, 'It's all set up for you' (...) The nurse came right away and said to [name of son], 'We have your hoist ready, it's been charged, we knew you were coming"

Carer (questionnaire): 'A member of staff came in to relieve us during the day for us to go and get drinks, food etc. This is vital support to us as our son will pull on and grab any tubes etc. So you can see how important it is to have someone with him at all times'

Carer (interview): 'I told them he always has problems with diarrhoea when he is on antibiotics (...) They didn't take any notice of what I said, and they gave him the antibiotics and he had diarrhoea for 12 days'

Consultant physician (interview): '[The patient] had cancer and needed surgery. I didn't realise he didn't have capacity to say 'no' to the operation. He didn't want the operation, and I just thought that was that. But [IDLN] came along and asked him, 'What do you think will happen if you don't have the operation?' and he really didn't know. He didn't have the capacity. So it became a 'best interest' decision, and we decided to do the operation'

Person with intellectual disabilities (interview): 'The only time that went well is when I saw the [IDLN].'

Carer (interview): '[IDLN] has done a lot of work and I have seen the difference. Dealing with the staff, receptionists, doctors, they are more understanding of [my daughter]'s needs' to make reasonable adjustments often lay on a spectrum ranging from 'common sense' to 'breaking the rules'. Wards that were perceived as 'good' by carers and staff appeared to have a culture where staff felt confident and able to make reasonable adjustments, with the support and supervision of senior colleagues. Conversely, people with additional support needs were not readily accommodated in some wards, and this appeared to be related to a ward culture where staff on all levels were unable to adjust their services. This could place a strain on carers:
Family carer (interview): 'I felt I couldn't leave him. I stayed with him from $8 \mathrm{am}$ to $7 \mathrm{pm}$ every day, because the nurses were so clueless.'

One of the six hospitals in the study was moving away from flagging specific vulnerable patient groups and was in the process of introducing a system where it was the need for specific reasonable adjustments, rather than the patient's vulnerability, that was flagged. There was as yet no feedback on the use or effectiveness of this system. 
Poor staff understanding of the needs of patients who have intellectual disabilities may be related to a lack of training. In response to the question whether staff had received training or guidance to help them to care for this group of patients, $35 \%$ of medical/dental staff $(n=139), 29 \%$ of registered nurses $(n=454)$ and $57 \%$ of healthcare assistants $(n=56)$ indicated on the questionnaire that they had never received any such training.

\section{Lack of clear lines of responsibility for implementing reasonable adjustments}

Those with specific responsibility for ensuring that patients with intellectual disabilities received reasonably adjusted healthcare varied across study sites and included the IDLNs, the trust's clinical lead for safeguarding vulnerable adults and the hospital matrons. However, at ward or clinic level, there was generally no clear allocation of responsibility or accountability for ensuring that each patient had his or her care and treatment needs met, and there was a lack of continuity of care. Carers reported seeing different members of staff on each shift. This led to good practice being dependent on individual staff members' attitudes and understanding, and the implementation of reasonable adjustments was patchy.

\section{Lack of allocation of additional funding and resources}

Many reasonable adjustments require resources in terms of time and money, in particular for adjustments that involve a provision of extra aids or services. At present, NHS acute trusts do not receive additional funds for providing services to patients who have intellectual disabilities through the national 'Payment by Results' system, nor through local commissioning. This was particularly noted by ward managers and matrons, who had responsibilities for allocating resources on ward level:

Ward manager (interview): 'If I've got patients who [need] ventilating so they need more one-to-one nursing care, I can increase my nursing team. If I've got patients who are high risk of falls I can increase my nursing team (...) but at the moment there is no tag for learning difficulties to increase your numbers.'

Matron (panel discussion): We're putting in additional resources to make the reasonable adjustments but actually we're not seeing any support in financial terms for that.'

\section{Enablers of providing reasonable adjustments}

The presence of an IDLN within the hospital emerged as the most significant enabler for the provision of reasonably adjusted hospital services. Ward managers were also important as potential enablers.

\section{Intellectual Disability Liaison Nurses}

IDLNs have a specific remit to improve hospital care for patients with intellectual disabilities. It was often difficult for ward staff to know what specific adjustments might be needed by patients with intellectual disabilities. These adjustments could be so complex that the support of a dedicated, highly skilled IDLN service was a key element in ensuring that preventable harm and preventable deaths were avoided in a number of cases. Their input was highly valued by carers and staff alike:

Family carer (interview): '[IDLN] went in there frequently, I got the impression at least once a day (...) She understood many of the issues that [my sister] had and was able to liaise.'

Ward staff nurse: 'Without [IDLN input] there is no sort of yardstick or benchmark. You know, a phone call: "Have you done this? Have you done that?" Even if it's "yes, yes, yes, yes", that's fine-but if it's "no, no, no, no", then she can either help, or give advice.'

However, for the IDLN role to be effective, staff needed to have easy access to the postholder, and the IDLN needed to have sufficient authority as well as management support.

\section{Ward managers}

The ward manager or sister had a strong influence on the presence or absence of a positive ward culture that could cater flexibly for individual patient needs. The following quotes from carers, one positive and one negative, demonstrate this:

Interviewer: What do you think makes the staff so good on that ward?'

Carer: I put it down to the ward manager. It affects the total attitude of all the staff.'

Carer (interview): 'Staff just didn't seem interested [in the patient]. The ward manager was not interested either. It was like she was just the manager in name, sitting in the office, and under her was a multitude of ward sisters all doing their own thing.

\section{DISCUSSION}

Patients with intellectual disabilities may need wide ranging and highly individual adjustments to healthcare services to ensure that they are not disadvantaged when accessing these services. Within the hospital trusts, there was evidence of flexible, lateral thinking. However, their consistent implementation was often dependent on the knowledge, understanding and flexibility of individual staff and teams, leading to the delivery of reasonable adjustments being haphazard throughout the hospitals and clinical areas. The kind of adjustments needed by an individual patient with intellectual disabilities were not always identified, understood or supported by hospital staff in this study. There was a lack of effective systems for identifying the patient's vulnerability (intellectual disability) as well as the patient's individual need for adjustments to the delivery of care and treatment, 
and a lack of clear lines of responsibility for implementing reasonably adjusted care to individual patients.

The differences between the conceptual framework (derived from the literature) and the empirical framework are mainly ones of degrees. Items added to the analytical framework during the course of the study were predominantly around staff understanding and staff responses. While there has been a great deal of emphasis on having the right policies and procedures in place, this research shows that the response of individual staff members, leadership, ward culture and resources are more important.

These issues are not unique to patients with intellectual disabilities. The skills required for recognising the need for reasonable adjustments and implementing them are likely to be transferrable to all vulnerable patient groups. An audit of dementia care in 55 general hospitals in England ${ }^{20}$ found that there was a general lack of patient-centred care, with environments that were not dementia-friendly. Pockets of good practice were identified but there was no evidence of effective organisational systems for implementing patient-centred care. An inquiry into the high numbers of deaths at an NHS hospital trust in England $^{21}$ highlighted a poor culture and lack of patient-centred care leading to severely compromised patient safety, with vulnerable elderly patients particularly at risk. The recommendations included better ward leadership and clear lines of responsibility for each patient's care.

The newly introduced practice at one of the hospital trusts of flagging the need for specific reasonable adjustments deserves further attention, as it appears to circumvent the problems of correctly identifying and flagging vulnerabilities. It also addresses the fact that while some reasonable adjustments may be needed by many patients with intellectual disabilities (eg, 'support with communication' or 'needs a carer present'), they are not unique to this group of patients, nor are they needed by all patients with intellectual disabilities. Finally, such a system would address the consistently reported concern of clinical staff in this study that flagging intellectual disability may not necessarily lead to their specific needs being addressed, and that it is important to assess each patient's need individually-including those of patients with other kinds of vulnerabilities or impairments.

Barriers that will need to be addressed on a national level include the lack of effective systems for identifying patients with intellectual disabilities, a lack of funding for those reasonable adjustments that are costly, and the paucity of training on intellectual disability in clinicians' training programmes. This includes healthcare assistants, who are least likely to be trained yet have the most frequent contact with patients with intellectual disabilities. There also needs to be a clear allocation of responsibility and accountability for the care of individual vulnerable patients, as well as overall coordination of their often complex hospital care. The evidence from this study suggests that ward managers are a critical part of the line of responsibility and accountability for meeting the needs of patients with intellectual disabilities, as they have direct responsibility for the behaviour of frontline staff who are key to delivering reasonably adjusted health services at ward or clinic level.

\section{Research recommendations}

Further insights are needed into the types of reasonable adjustments that patients with intellectual disabilities, as well as patients with other kinds of vulnerabilities, need most during their hospital pathways, and what the resource implications for this are. This knowledge is important in order to identify where NHS resources should be directed. The Intellectual Disability Observatory is collecting evidence about the types of reasonable adjustments currently implemented by NHS services. ${ }^{13} 22$ However, the need for reasonable adjustments has not been quantified.

Further research is therefore warranted to answer the following questions: (A) What reasonable adjustments are needed most frequently within the hospital care pathways of vulnerable patient groups? (B) What knowledge, systems and structures are needed within the hospital to ensure that these reasonable adjustments are routinely made? $(\mathrm{C})$ What is the most effective training to prepare staff to provide individualised reasonably adjusted services and to use the Mental Capacity Act effectively? (D) What are the cost implications for reasonable adjustments? This question relates not only to the cost of implementing the reasonable adjustment, but also to the estimated cost savings in relation to, for example, lower re-admission rates, fewer missed appointments and shorter lengths of admission. Possible ways of translating this into appropriate cost provision should be considered (such as, eg, the Payment by Results system or through locally developed Commissioning for Quality and Innovation (CQUIN) targets).

Such research might take the form of a large-scale multiple case study where the hospital pathways of a large number of people with intellectual disabilities and other vulnerabilities are reviewed in-depth, identifying and quantifying the barriers they faced as well as the reasonable adjustments they needed.

\section{Conclusion}

Providing reasonably adjusted services for people with impairments is a legal requirement. Failure to make reasonable adjustments can lead to poorer outcomes. This study has confirmed findings from previous studies and inquiries that acute hospital trusts fail to deliver services that are consistently accessible to patients with intellectual disabilities, and has added evidence to the existing knowledge base about the reasons why this is the case. The iterative research process of interpretation and review in a mixed-methods study has been highly relevant; in particular, stakeholder interviews and strong user involvement were important. 
A senior clinical manager or ward manager should be accountable for the provision of reasonable adjusted health services, and should ensure that a named staff member (possibly the ward manager him/herself) is responsible for identifying the need for service adjustments of any patient entering the healthcare system. The accountable manager should also ensure that all staff understand these needs, and that the appropriate reasonable adjustments are delivered. The provision of such reasonable adjusted healthcare for people with intellectual disabilities could act as a yardstick for measuring the overall quality of any healthcare service.

\author{
Author affiliations \\ ${ }^{1}$ Faculty of Health, Social Care and Education, St George's University of \\ London and Kingston University, London, UK \\ ${ }^{2}$ Division of Population Health Sciences and Education, St George's, \\ University of London, London, UK \\ ${ }^{3}$ Institute of Leadership and Management in Health, Kingston University \\ Business School, Kingston, UK
}

Acknowledgements St George's University of London provided administrative support and support with IT, including the development of the e-Questionnaire; and provided staff support for the coresearchers with intellectual disabilities. Paul Adeline contributed to data collection tool development for people with intellectual disabilities, conducted interviews with people with intellectual disabilities and contributed to data interpretation in relation to people with intellectual disabilities. Jim Blair contributed to protocol development. Gary Butler contributed to data collection tool development for people with intellectual disabilities, conducted interviews with people with intellectual disabilities and contributed to data interpretation in relation to people with intellectual disabilities. Sara Christian contributed to staff questionnaire development. Leonard Fenwick chaired the Research Advisory Board and contributed to study design and interpretation of data. Vanessa Gordon contributed to interpretation of data around patient safety and incident reports. Lloyd Page contributed to data collection tool development for people with intellectual disabilities. Vicky Raphael contributed to data interpretation in relation to carers. Monica Stannard contributed to data collection tool development for carers and data interpretation in relation to carers. Members of the research advisory board provided project governance and contributed to interpretation of data. In particular, and in addition to the contributors listed above: Vikki Carruth, Amanda Cresswell, Alison Robertson.

Contributors IT-W was the prinicipal investigator of the study. She had overall management and supervision of the project; was the major contributor to protocol development, study design and data collection tool development; supervised, and contributed to, data collection; contributed to data analysis; was the main contributor to data interpretation and synthesis; and drafted the manuscript. LG contributed to data collection tool development; carried out data collection, data analysis and data interpretation; contributed to drafting the manuscript and critically revised the manuscript. NG contributed to protocol development, study design and data collection tool development; carried out data collection and data analysis; contributed to data interpretation; and critically revised the manuscript. EA contributed to data collection tool development; carried out data collection and data analysis; contributed to data interpretation; and critically revised the manuscript. SG contributed to protocol development and study design; advised on qualitative methodological issues and project management; and critically revised the manuscript. SW contributed to survey development and data analysis; advised on quantitative methodological issues; and critically revised the manuscript CE contributed to protocol development, study design and interpretation of data; and critically revised the manuscript. SH identified the need for the project; supervised the principal investigator; contributed to protocol development, study design and interpretation of data and critically revised the manuscript.
Funding This manuscript presents independent research commissioned and funded by the National Institute for Health Research (NIHR) Health Services and Delivery Research (HS\&DR) Programme, grant number 10/1007/22. The NIHR reviewed the protocol, monitored progress, and reviewed the findings.

\section{Competing interests None.}

Ethics approval The study was approved by the National Research Ethics Services (NRES, reference 11/L0/0428). Local research approvals were obtained at each participating hospital trust.

Provenance and peer review Not commissioned; externally peer reviewed.

Data sharing statement Extended description of methodology and extra data are available. $^{18}$

Open Access This is an Open Access article distributed in accordance with the Creative Commons Attribution Non Commercial (CC BY-NC 3.0) license, which permits others to distribute, remix, adapt, build upon this work noncommercially, and license their derivative works on different terms, provided the original work is properly cited and the use is non-commercial. See: http:// creativecommons.org/licenses/by-nc/3.0/

\section{REFERENCES}

1. Emerson E, Hatton C. People with learning disabilities in England. London: Public Health England, 2008.

2. Heslop P, Blair P, Fleming P, et al. Confidential Inquiry into premature deaths of people with learning disabilities (CIPOLD): final report. Bristol: Norah Fry Research Centre, 2013.

3. Emerson E, Baines S. Health inequalities and people with learning disabilities in the UK. Tizard Learn Disab Rev 2011;16:42-8.

4. Michael J. Healthcare for all: report of the independent inquiry into access to healthcare for people with learning disabilities. London: Aldridge Press, 2008.

5. MENCAP. Death by indifference. London: Mencap, 2007.

6. MENCAP. Death by indifference: 74 deaths and counting. A progress report 5 years on. London: Mencap, 2012.

7. Parliamentary and Health Service Ombudsman. Six lives: the provision of public services to people with learning disabilities. HC 203 I-VIII. London: The Stationery Office, 2009.

8. Department of Health. Six lives progress report. London: Department of Health, 2010.

9. Hollins S, Tuffrey-Wijne I. Meeting the needs of patients with learning disabilities. BMJ 2013;346:f3421.

10. HMSO. Disability Discrimination Act 2005. London: HMSO, 2005.

11. HMSO. Equality Act 2010. London: HMSO, 2010.

12. Equality and Human Rights Commission. Your rights to equality from health and social care services. Equality Act 2010 guidance of your rights, volume 5 of 9. Manchester: EHRC, 2010. 18 Dec 2012.

13. Hatton $\mathrm{C}$, Roberts $\mathrm{H}$, Baines $\mathrm{S}$. Reasonable adjustments for people with learning disabilities in England 2010: a national survey of NHS Trusts. Improving Health and Lives: Learning Disabilities Observatory, 2011.

14. Disability Rights Commission. Equal treatment: closing the gap-a formal investigation into physical health inequalities experienced by people with learning disabilities and/or mental health problems. London: Disability Rights Commission, 2006.

15. Disability Rights Commission. The duty to promote equality: statutory code of practice England and Wales. London: HMSO, 2005.

16. MENCAP. Getting it right: a campaign guide. London: Mencap, 2010.

17. Department of Health. Health inequalities: progress and next steps. London: The Stationery Office, 2008.

18. Tuffrey-Wijne I, Giatras N, Goulding L, et al. Identifying the factors affecting the implementation of strategies to promote a safer environment for patients with learning disabilities in NHS hospitals: a mixed-methods study. Health Serv Deliv Res 2013;1-224. doi: 10.3310/hsdr01130

19. Department for Constitutional Affairs. Mental Capacity Act 2005: code of practice. London: The Stationery Office, 2007.

20. Young J, Hood C, Woolley R, et al. Report of the national audit of dementia care in general hospitals 2011. London: Royal College of Psychiatrists, 2011.

21. Francis R. The report of the Mid Staffordshire NHS Foundation Trust Public Inquiry-HC947. London: The Stationery Office, 2013.

22. Improving Health and Lives (IHAL). Reasonable adjustments database. 2013. http://www.improvinghealthandlives.org.uk/ adjustments (accessed 19 Nov 2013). 